\title{
Multivariate Statistical Analysis of STEM-EDS at the Noise Floor
}

\author{
C. M. Parish, P. D. Edmondson, and M. K. Miller \\ Oak Ridge National Laboratory, PO Box 2008, MS 6064, Oak Ridge, TN 37831-6064
}

Multivariate statistical analysis (MVSA) methods, such as principal component analysis (PCA), are often used to aid the qualitative interpretation of spectrum imaging (SI) experiments or to perform noise filtering prior to pixel-by-pixel quantification. MVSA methods provide excellent discrimination of low volume-fraction phases, down to a single pixel level in the presence of a sufficiently high signal-to-noise ratio [1]. However, energy dispersive spectrometry (EDS) in scanning transmission electron microscopy (STEM) experiments at nm-resolution can result in low signal levels. This paper studies the effects of low signal levels on the performance of optimally scaled PCA methods [2] on STEM-EDS spectrum images for a complex multiphase material.

The material studied is a nanostructured 14YWT ferritic alloy (NFA) with a nominal composition of Fe-14.2 wt. \% Cr- $1.95 \% \mathrm{~W}-0.22 \% \mathrm{Ti}-0.25 \%+\mathrm{Y}_{2} \mathrm{O}_{3}$ that is intended for nuclear applications. The material consists of Ti-Y-O nanoclusters $(\mathrm{NCs})$ and $\mathrm{Cr}-\mathrm{W}$ enriched grain boundaries in an $\mathrm{Fe}-\mathrm{Cr}-\mathrm{W}$ matrix. X-ray count maps for $\mathrm{Fe}, \mathrm{Cr}, \mathrm{Y}$, and Ti-K $\alpha$ and $\mathrm{W}-\mathrm{L} \alpha$, as well as a Ti- $\mathrm{L}_{2,3}$ EELS map, are shown in Fig. 1. EDS and EELS spectrum images were acquired simultaneously on a Philips CM200 FEG-STEM using Gatan Digiscan II hardware and software, an EDAX Si(Li) EDS detector at $10 \mathrm{eV} /$ chan with $51.2 \mu \mathrm{s}$ amp time, and a Gatan Imaging Filter (GIF) 200 at $0.5 \mathrm{eV} /$ chan and 16× vertical binning for EELS. Data are $40 \times 40$ pixels over $80 \times 80 \mathrm{~nm}$ with $500 \mathrm{~ms}$ dwell and a $\sim 1 \mathrm{nA}$, $\sim 1.5 \mathrm{~nm}$ probe. The specimen was $\mathrm{Ga}^{+}$focused ion beam (FIB) taper-cut followed by low-voltage $\mathrm{Ar}^{+}$cleaning; the final specimen thickness was $\sim 0.3 \mathrm{t} / \lambda$, or $\sim 40-60 \mathrm{~nm}$. Cr-W enrichment is visible at the grain boundaries and EELS shows Ti-rich precipitates at the boundaries. The Ti and Y EDS are very noisy. Poisson-scaled PCA with $2^{2}$ spatial binning and up-sampling indicates an algebraic rank of 2, Fig. 2 (Mo is from the FIB lift-out support). Inspection of the EDS and EELS maps shows the sample's chemical rank is at least 3 (matrix, grain boundary enrichment, Ti-rich precipitates). However, since the signal level is so low, the PCA algorithm incorrectly convolved the precipitate and grain boundary contributions into a single eigenvector. The count maps, Fig. 3, were calculated identically to those in Fig. 1 except a PCA rank-2 model was used instead of the raw X-ray counts. The PCA-derived maps (Fig. 3) do not agree with the raw maps (Fig. 1), particularly the PCA TiEDS map compared to the raw Ti-EELS map. It is inferred that when signal/noise is too low, the PCA routine finds a statistically succinct but metallurgically incorrect model of the physical situation. To test this, DTSA-II software [3] was used to simulate the EDS data for Fe-14Cr-2W (matrix), $\mathrm{Fe}-20 \mathrm{Cr}-5 \mathrm{~W}$ (grain boundary), and $\mathrm{Ti}_{2} \mathrm{Y}_{2} \mathrm{O}_{7}$ (precipitate) phases, which were arranged into a simulated EDS SI with different Poisson noise added at levels of 500 or 5,000 mean X-rays/pixel (500 counts/pixel is approximately equal to the experimental data). At 500 counts/pixel, the simulation gives the same incorrect rank-2 PCA result as in the experiment. However, with a 10× higher signal, PCA provides an algebraic rank-3 solution, correctly matching the chemical rank, Fig. 4. Thus, when multiple phases are present and the signal/noise level is very low, it is important to use additional information (such as co-acquired EELS SI) to check the accuracy of MVSA results, thus ensuring that spurious interpretations are not reached. 
[1] P.G. Kotula et al., Micros. Microanal., 9 (2003) 1; C.M. Parish et al., J. Am. Ceram. Soc., 91(2008) 3690; M.G. Burke et al., J. Mater. Sci., 41(2006) 4512.

[2] M. R. Keenan \& P. G. Kotula, Surf. Int. Anal., 36(2004) 203.

[3] N. W. M. Ritchie, Micros. Microanal., 15(2009) 454.

[4] Research sponsored by the Office of Basic Energy Sciences, Materials Science and Engineering Division, U.S. Department of Energy, and by ORNL's Shared Research Equipment (SHaRE) User Facility, which is sponsored by the Office of Basic Energy Sciences, U.S. Department of Energy.

\begin{tabular}{|c|c|c|c|c|c|}
\hline Fe-K $\alpha$ EDS & Cr-K $\alpha$ EDS & W-L $\alpha$ EDS & Y-K $\alpha$ EDS & Ti-K $\alpha$ EDS & Ti-L2,3 EELS \\
\hline 3. & 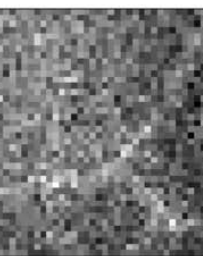 & 5 & 8 & 48 & $\frac{4}{42}$ \\
\hline
\end{tabular}

FIG. 1. EDS maps for Fe, Cr, W, Y, and Ti, and EELS map for Ti.

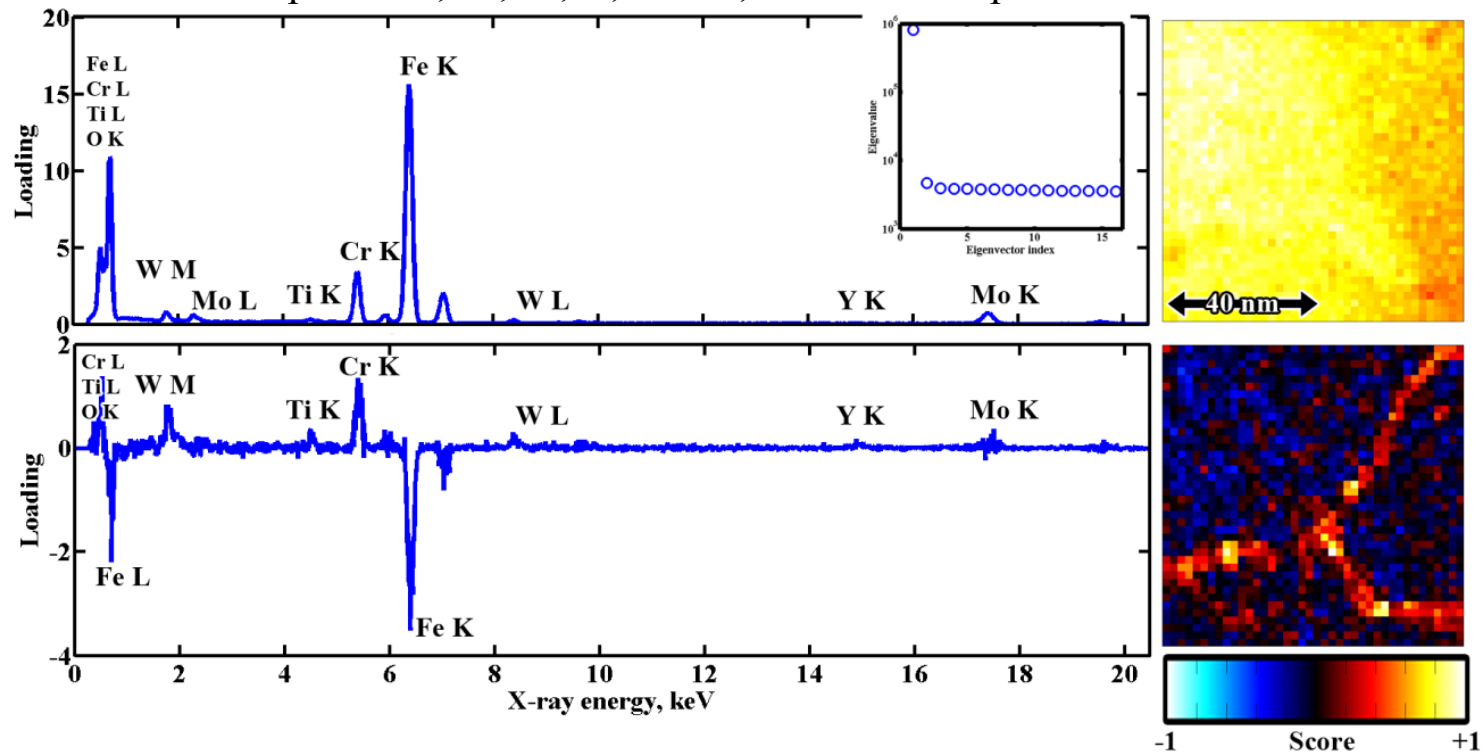

FIG. 2. PCA analysis of the EDS-SI. Top row is PC-1, bottom row PC-2, and inset the scree plot.
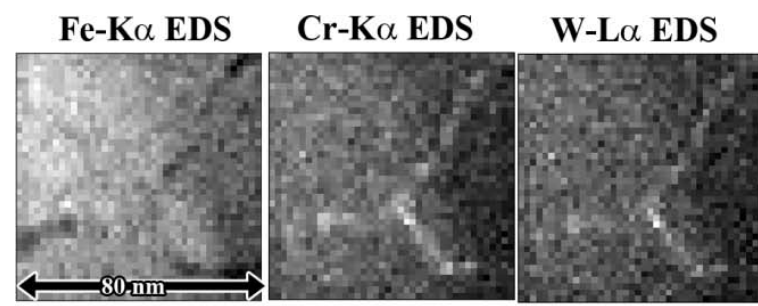

Y-K $\alpha$ EDS Ti-K $\alpha$ EDS
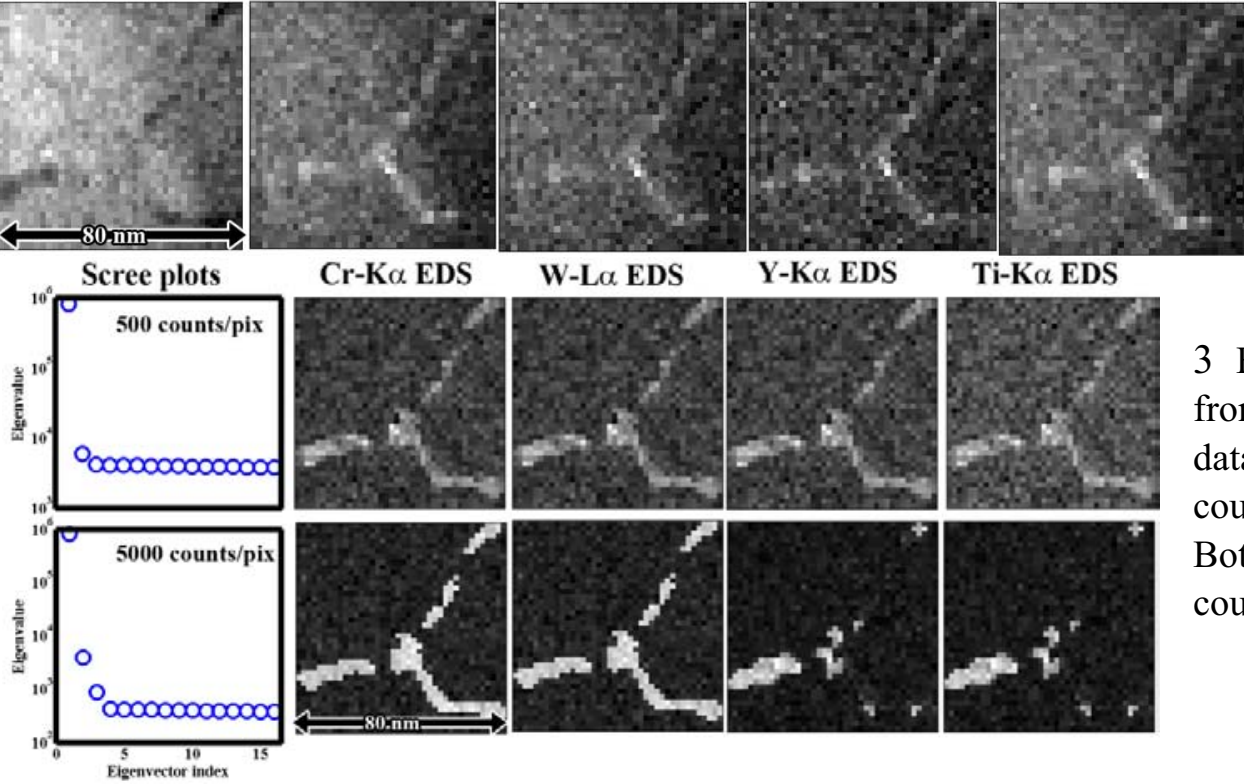

FIG. 3: EDS maps derived from the rank-2 PCA model. 